inhaled exposures at an individual level, and the attitudes of workers with and without COPD to these issues.

Aim The aim of this work was to explore attitudes to workplaces, and to other aspects of the management of long-term respiratory problems, from individuals within a large population study with and without COPD.

Methods The primary aim of this population-based study was to assess the contribution made by inhaled occupational exposures to the development of COPD. The study was based in Sheffield, historically an industrialised part of the UK. A sub sample of cases of self reported COPD $(n=66)$ and non cases of COPD $(n=224)$ were asked to rate their views to a set of 36 pre defined statements, each rated between "don't agree" and "completely agree" on a five point scale. Statements included enquiry about attitudes to chronic respiratory ill health, smoking, general health issues and the influences of the workplace on health.

Results 290 individuals, all 55 years old or greater, participated, 172 (59\%) of whom were male. The majority of participants generally agreed or completely agreed with most statements, although various differences emerged between those with and without COPD. For example, those with self reported COPD were more likely, as anticipated, to identify this condition as a longer term health problem, but less likely to agree that workers with possible breathing problems should talk to their employer about these or undergo regular spirometry to identify these.

Conclusions This study has identified a set of attitudes and beliefs from those with and without COPD relating to chronic respiratory problems at work. Knowledge of these semi-quantitative data will assist the development of better workplace interventions to reduce the burden of this condition.

\section{P128 A NEW, EFFICIENT SPIROMETRY-BASED ALGORITHM TO PREDICT RESTRICTIVE LUNG DISEASE IN WORKPLACE RESPIRATORY SURVEILLANCE}

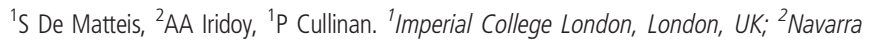
Hospital Complex, Pamplona, Spain

\subsection{6/thoraxjnl-2014-206260.269}

Introduction and objectives Spirometry is frequently carried out as part of workplace-based respiratory surveillance programmes for the detection of both obstructive and restrictive lung diseases. However, the performance of spirometry to detect restrictive lung diseases is generally poor and especially so if the prevalence of the disease in the tested population is low such as in many working populations.

Our aim was to increase the specificity and the positive predictive value (PPV) of current spirometry-based algorithms to diagnose restrictive lung diseases in the occupational health setting to reduce false positives and so the number of unnecessary and expensive referrals for lung volume measurements in hospital.

Methods We re-analysed two prospective studies of 259 and 265 tertiary care hospital consecutive patients, respectively used to derive and validate the current standard spirometry-based algorithm (FVC $<85 \%$ predicted and FEV1/FVC $>55 \%$ ) to diagnose restrictive lung diseases (Glady CA, et al. Chest 2003). We used true lung restrictive cases (TLC $<$ LLN predicted) as a gold standard in $2 \times 2$ contingency tables to estimate sensitivity, specificity, positive and negative predictive values for each potential diagnostic cut-off. Predicted values for spirometry parameters were calculated by using both Crapo and Hankinson equations. Because our target population is active workers we tested the performance of each diagnostic algorithm among subjects under
65 years old and with a simulated prevalence of restrictive disease of $10 \%$ and $1 \%$. In addition, we compared the performance of our best diagnostic algorithm to the ones previously reported by using receiver operating characteristic (ROC) curves.

Results Our best diagnostic algorithm (FVC $<70 \%$ predicted and FEV1/FVC $\geq 0.7$ ) had a higher specificity (96\% using Hankinson prediction equation) and PPV (80\% and $27 \%$ for a disease prevalence of $10 \%$ and $1 \%$, respectively) compared to previous algorithms. For example, compared to Glady's algorithm, among 184 people tested, ours produced only 6 (3\%) false positives vs. 64 (34\%), and correctly classified 91\% subjects vs. $65 \%$, corresponding to an area under the ROC curve of 0.83 vs. 0.77 . The results were confirmed in the validation dataset.

Conclusions Our proposed spirometry-based algorithm accurately excludes pulmonary restriction and reduces unnecessary lung volume testing in occupational health clinical setting.

\section{P129 SYSTEMATIC REVIEW AND META-ANALYSIS OF CROSS SECTIONAL STUDIES ON ARC WELDING FUME EFFECTS AND OBSTRUCTIVE LUNG DISEASE}

A Marongiu, C Minelli, C Canova, S Schofield, J Szram, P Cullinan. Department of Occupational and Environmental Medicine, NHLI, Imperial College, London

\subsection{6/thoraxjnl-2014-206260.270}

Introduction and objectives. Exposure to the complex metalrich aerosol produced by arc welding is putatively linked to obstructive lung disease in welders through a mechanism of oxidative stress. Given that published studies have been mainly conducted by means of cross-sectional surveys, this research aims systematically to review these; we have previously published a similar review of (the many fewer) longitudinal studies on welders' lung function decline.

Methods Medline, Embase and Web of Science were searched up to July 2013 by combining two blocks for exposure (welding fume) and outcomes $\left(\mathrm{FEV}_{1}, \mathrm{FVC}, \mathrm{FEV}_{1} / \mathrm{FVC} \%\right.$, asthma, acute and chronic bronchitis, dyspnoea and wheezing) both containing free text keywords and MeSH terms. Double data extraction was performed independently. To explore possible causes of heterogeneity a subgroup analysis was undertaken to account for country income level, participants' smoking status and welders' age.

Results Fifty-six cross-sectional studies were identified and 40 were included in the review. Of these, 35 were included in a meta-analysis; lung function was measured in 22 studies and symptoms in 30 studies. Compared with non-welders, welding was associated with a statistically significant reduction in $\mathrm{FEV}_{1}$ $\left(\mathrm{SMD}=-0.34 ; 95 \% \mathrm{CI},-0.57,-0.12 ; \mathrm{I}^{2}=92 \%\right)$ and $\mathrm{FEV}_{1} / \mathrm{FVC} \%$ $\left(\mathrm{SMD}=-0.51 ; 95 \% \mathrm{CI},-0.94,-0.08 ; \mathrm{I}^{2}=96 \%\right)$. The substantial heterogeneity in both outcomes was explored further but no subgroup analyses explained it. FVC reduction with welding exposure was not statistically significant and highly heterogeneous. Welding was associated with asthma $(\mathrm{OR}=1.65,95 \% \mathrm{CI}$, $\left.1.14,2.37 ; \mathrm{I}^{2}=0 \%\right)$, acute bronchitis $(\mathrm{OR}=1.61,95 \% \mathrm{CI}, 1.15$, $\left.2.27 ; \mathrm{I}^{2}=11 \%\right)$, chronic bronchitis after excluding a large study ${ }^{1}$ $\left(1.92,95 \%\right.$ CI, 1.50, 2.46; $\left.\mathrm{I}^{2}=40 \%\right)$, dyspnoea in medium/low income countries $\left(\mathrm{OR}=3.54,95 \% \mathrm{CI}, 1.96,5.08 ; \mathrm{I}^{2}=0 \%\right)$ and wheezing among non-smokers $(\mathrm{OR}=9.06,95 \% \mathrm{CI}, 3.75,21.9$; $\left.\mathrm{I}^{2}=0 \%\right)$. All risk estimates for symptoms were higher in medium/low income countries.

Conclusions Welding fume exposure has a negative effect on the respiratory health of workers, possibly leading to obstructive lung disease and increasing the risk of respiratory symptoms particularly in medium/low income countries. 


\section{REFERENCE}

1 Groth $M$, Lyngenbo 0 . Respiratory symptoms in Danish welders. Scand J Soc Med, 1989;17(4):p. 271-6

\section{P130 FEASIBILITY STUDY OF A PRIMARY CARE SCREENING TOOL FOR OCCUPATIONAL ASTHMA}

GI Walters, DA Fitzmaurice, JG Ayres. University of Birmingham, Birmingham, UK

\subsection{6/thoraxjnl-2014-206260.271}

Introduction Occupational asthma (OA) accounts for 1 in 6 cases of adult-onset asthma and is associated with an estimated societal cost in the UK of $£ 100$ million/annum. ${ }^{1}$ However many workers with OA go undiagnosed or experience a lengthy delay in diagnosis, ${ }^{2}$ and primary healthcare professionals fail to enquire about patients' occupations and the effect of work on asthma symptoms. ${ }^{3}$ We evaluated the feasibility of introducing an electronic screening tool for OA in primary care.

Methods A prospective feasibility study was undertaken over a 3month period in 4 primary care practices in Birmingham, UK. Practices modified their existing electronic health records (one of: EMIS, SystmOne, Vision) with a customised asthma review template embedding the questions "What is your occupation?" and "Are your symptoms better away from work on days away/on holiday?" Baseline practice-level data were gathered and at the end of the study period all exposed healthcare professionals (GPs, practice nurses) were invited to complete an online questionnaire intended to evaluate utility and willingness to use the tool.

Results Prevalence of Read-coded asthma was 5.6-8.2\% and Read-coded OA was $0-0.7 \%$. All 4 practices incorporated the screening tool without any technical difficulty. 24/52 (46\%) exposed GPs/nurses returned questionnaires, of whom 10 (42\%) had used the tool; uptake was higher (85\%) in those professionals who were given brief training. Healthcare professionals who did use the screening tool found it to be user-friendly (clear, concise, logical) with no perceived procedural or IT difficulties or significant added burden. Responders were less confident (44\% agreed/strongly agreed) about how to act when patients had work-related asthma symptoms and 78\% agreed/strongly agreed that further training in managing health aspects of suspected occupational asthma would improve the screening tool.

Conclusion An electronic screening tool for OA can be easily and quickly incorporated into existing asthma disease management systems. Its utility could be greatly improved by user instruction and training in further clinical management of the patient with work related asthma symptoms.

\section{REFERENCES}

1 Ayres et al. Thorax 2011;66(2):128-33

2 Fishwick et al. PRCJ 2007:16:304-10

3 Walters et al. Occup Med 2012:62:570-3

\section{P131 UNDERSTANDING HEALTH BELIEFS AND BEHAVIOUR IN WORKERS WITH OCCUPATIONAL ASTHMA}

${ }^{1} \mathrm{GI}$ Walters, ${ }^{2}$ AS Robertson, ${ }^{2}$ PS Burge, ${ }^{1} \mathrm{JG}$ Ayres, ${ }^{1}$ AS Soundy. ${ }^{1}$ University of Birmingham, Birmingham, UK; ${ }^{2}$ Heart of England NHS Foundation Trust, Birmingham, UK

\subsection{6/thoraxjnl-2014-206260.272}

Introduction Long delays from symptom onset to the diagnosis of occupational asthma have been reported in the UK, Europe and Canada and workers are often reluctant to seek medical help or workplace solutions for their symptoms. ${ }^{1,2}$ Reducing this delay could improve workers' quality of life, and reduce the societal cost of occupational asthma. This study aimed to explore reasons behind such delays.

Methods A purposive sample of 20 individuals diagnosed with, or under investigation for, occupational asthma (median age $=52 ; 70 \%$ male; $80 \%$ white British) undertook a single semistructured interview. Interviews were transcribed verbatim and thematic analysis was undertaken in order to explore health beliefs and identify barriers to diagnosis.

Results Four themes were identified: (1) workers' understanding of symptoms, (2) working relationships, (3) workers' course of action and (4) workers' negotiation with healthcare professionals. Understanding of symptoms varied between individuals, from a lack of insight into the onset, pattern and nature of symptoms, through to misunderstanding of what they represented, or ignorance of the existence of asthma as a disease entity. Workers described reluctance to discuss health issues with managers and peers, through fear of job loss and a perceived lack of ability to find a solution.

Conclusion The evolution of workers' understanding depended upon how actively they looked to define symptoms or seek a solution. Proactive workers were motivated to seek authoritative help and negotiate inadequate healthcare encounters with GPs. In summary there appear to be a number of key influences motivating a worker to seek an explanation for their symptoms or a definitive solution, which are represented in the model in Figure 1. Understanding workers' health beliefs will enable policy makers and clinicians to develop better workplace interventions for identifying occupational asthma.

\section{REFERENCES}

1 Fishwick D et al. Prim Care Respir J 2007;16: 304-10

2 Poonai N et al. Can J Public Health 2005:96:230-3

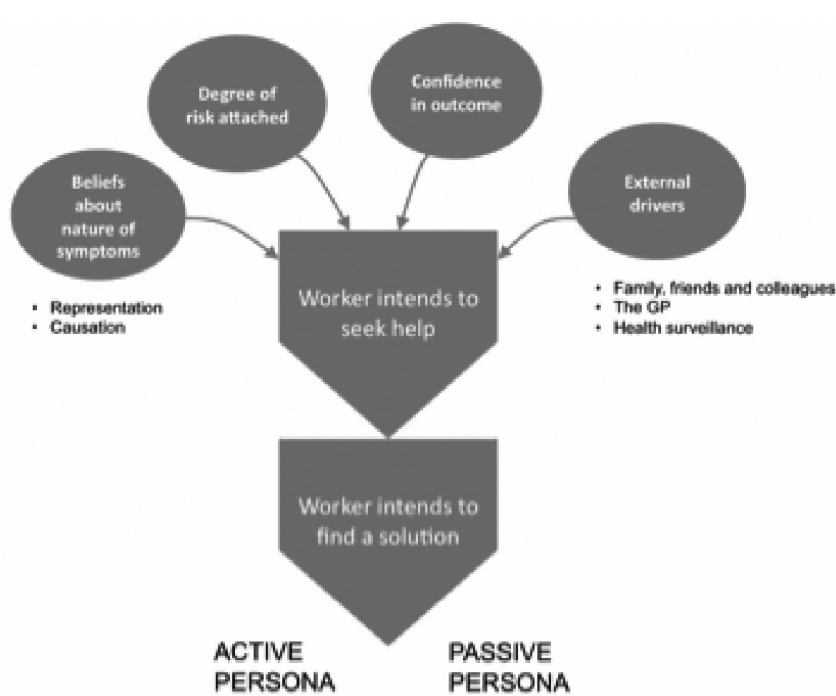

Abstract P131 Figure 1 The major influences on workers' healthseeking behaviour

\section{P132 CLEANER'S ASTHMA : NOW YOU SEE IT, NOW YOU DON'T}

S Alfajjam, D Howel, C Stenton. University of Newcastle Upon Tyne, Newcastle Upon Tyne, UK

10.1136/thoraxjnl-2014-206260.273 